International Journal of Pure and Applied Mathematics

Volume 114 No. $3 \quad 2017,473-482$

ISSN: 1311-8080 (printed version); ISSN: 1314-3395 (on-line version)

url: http://www.ijpam.eu

doi: 10.12732/ijpam.v114i3.5

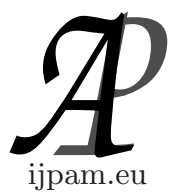

\title{
A SYSTEM OF PLANAR PIECEWISE ISOMETRIC SYSTEMS AND CODINGS OF THESE SYSTEMS
}

\author{
M.R. Sharifipour ${ }^{1 \S}$, H.M. Mohammadinejad ${ }^{2}$ \\ ${ }^{1,2}$ Department of Mathematics \\ Faculty of Mathematics and Statistics \\ University of Birjand, Birjand, IRAN
}

\begin{abstract}
In this paper we investigate dynamics of a system of planar piecewise isometric systems. At first we introduce a coding map on a system of planar piecewise isometric systems then we extend the coding map onto the entire phase space and prove that there exist an essential coding for a singular point. At the end of this paper we investigate some important sets including singular sets, exceptional sets, rational sets, etc... generated by a system of planar piecewise isometric systems.
\end{abstract}

AMS Subject Classification: 58F03, 52A07

Key Words: piecewise isometric, singular set, exceptional set, coding and essential coding, rational and irrational code, partition function

\section{Introduction}

In contrast to continuous dynamical systems, there has not been yet a systematic theory and only limited methods available in the study of dynamical system with discontinuities. But symbolic dynamics may be a useful tool in this study. For example, in [6, 28] symbolic dynamics analysis for generalized piecewise isometries has given. Piecewise isometries show a rich rang of dynamical behaviors under iteration $[2,4,5,8,32]$ and are a natural extension of the

Received: December 1, 2016

Revised: January 29, 2017

Published: $\quad$ May 23, 2017

$\S_{\text {Correspondence author }}$ (c) 2017 Academic Publications, Ltd. url: www.acadpubl.eu 
interval exchange to a class of Euclidean two-dimensional piecewise isometries $[11,13,22,24,25,34,35]$.

A somewhat unusual application has found outside of mathematics. In field of electronic engineering, in particular in the theory of digital filters $[1,15,16$ $, 17,23,29,31]$ also this kind of discontinuity systems appear in a variety of contexts like, interval translation [21], rectangular exchange [12], polygonal and polyhedron exchange [9] and billiards [7, 10].

In[2] developed a framework which classify planar piecewise isometries of a polygonal region of the plane with polygonal partition. In [3] the author begined underlying basic foundation of the theory of Euclidean piecewise isometric dynamical systems. The aim of this theory is study the long term behavior of state in a dynamical system which the local generating maps are isometries. In $[26,30]$ for planar piecewise isometries P.Ashwin has introduced symbolic codings underlying map operation and it has been shown that this kind of codings is helpful for revealing the dynamical properties of the map. In $[18,20]$ the stability of periodic points of piecewise isometries defind on the Euclidean space has investigated and in [14] proved that piecewise isometries in arbitrary dymensional Euclidean space have zero topologica entropy. In [19, 33] confirmed the existence of essntial coding for a forward singular point and present a necessary and sufficant condition for the existence of a admissible rational coding for a planar piecewise isometric map.

In this paper for a one-parameter family of planar piecewise isometries we will introduce symbilic codings underlying a one-parameter family of map operations and confirm that there must exist an essential coding for a forward singular point.

\section{Preliminary}

Let $\mathrm{M}$ be a subset of $\mathbb{R}^{n}$ and denote a colection of open subset of $\pi(M)$ (The phase space) by $M=\left\{M_{1}, \ldots, M_{m}\right\}$ where $\pi(M)=\bigcup_{i=1}^{m} \bar{M}_{i}$.

A pair (f,M) is called a piecewise isometry if,

I: $\quad M_{i} \cap M_{j}=\emptyset \quad$ For $\quad i \neq j \quad, i, j \in\{1, \ldots, m\}$, and each $M_{i}$ is called a partition atom of $\pi(M)$,

II: The restriction $\left.f\right|_{\bar{M}_{i}}$ to each partition atom $M_{i}, \mathrm{i}=1, \ldots, \mathrm{m}$ is an isometric map, i.e., $\left.f\right|_{\bar{M}_{i}}=f_{i}$ where $\left\{f_{1}, \ldots, f_{m}\right\}$ is a collection of isometric maps. 
At the same time, for any point $\mathrm{z}$ on the discontiniuity line $\partial M=\bigcup_{i \neq j}\left(M_{i} \cap M_{j}\right)$ (boundry of phase space), we prescribe that its iteration is multi-valued, i.e., we take both $f(z)=f_{i}(z)$ and $f(z)=f_{j}(z)$.

The piecewise isometries define in different ways have the same dynamical behavior. In this paper, we will focus on invertible up to a zero measure set.We further assume that all partition atoms $M_{k}(\mathrm{k}=1, \ldots, \mathrm{m})$ are convex planar polygons. Let

$$
\mathcal{E}_{-}(n)=\bigcup_{k=0}^{n} f^{-k}(\partial M) \quad \mathcal{E}_{+}(n)=\bigcup_{k=1}^{n} f^{k}(\partial M)
$$

we call $\mathcal{E}_{-}=\lim _{n \rightarrow \infty} \mathcal{E}_{-}(n)\left(\mathcal{E}_{-}=\bigcup_{k=0}^{\infty} f^{-k}(\partial M)\right)$ the forward singular set, and $\mathcal{E}_{+}=\lim _{n \rightarrow \infty} \mathcal{E}_{+}(n)\left(\mathcal{E}_{+}=\bigcup_{k=0}^{\infty} f^{k}(\partial M)\right)$ the backward singular set, and $\mathcal{E}=\mathcal{E}_{-} \cup \mathcal{E}_{+}$the singular set. At the same time, we define the exceptional set $\mathcal{F}$ as follows,

$$
\mathcal{F}=\left\{z \in M \mid i n f_{n \in \mathbb{Z}} d\left(f^{n}(z), \partial M\right)=0\right\}
$$

where $\mathrm{d}(.,$.$) represents the Euclidean metric. Obviously, the singular set is$ subset of exceptional set, i.e., $\mathcal{E} \subseteq \mathcal{F}$.

The singular set plays an important role in a piecewise isometric system, and it determines some basic dynamical properties of the system.

Given a piecewise isometry (f,M) with partition $M=\left\{M_{1}, \ldots, M_{m}\right\}$ and a sequence of maps $f=\left\{f_{1}, \ldots, f_{m}\right\}$, denoting the set of all infinite words over the alphabet $\{1, \ldots, m\}$ by $\sum(m)$. We define a coding map $\mathcal{L}: \pi(M) \backslash \mathcal{E}_{-} \mapsto$ $\sum(m)=\{1, \ldots, m\}^{\mathbb{N}}$ as

$$
\mathcal{L}(z)=\alpha=\alpha_{0} \alpha_{1} \ldots \text { where }[\mathcal{L}(z)]_{i}=\alpha_{i}=k \text { if } f^{i}(z) \in M_{k}
$$

. We call an infinite sequence $\alpha=\alpha_{0} \alpha_{1} \ldots$ admissible under map if there exists $z \in \pi(M) \backslash \mathcal{E}_{-}$such that $\mathcal{L}(z)=\alpha$, and $\alpha$ is called the coding of point $\mathrm{z}$. Similarly we say that a finite sequence $\beta=\beta_{0} \ldots \beta_{n-1}$ with length $\mathrm{n}$ is admissible, if there exists a non-forward-singular point $\mathrm{z}$ such that $\beta$ is pre word of $\mathcal{L}(z)$, i.e., $\mathcal{L}(z)=\beta \oplus \alpha$, and $\alpha$ is an admissible infinite sequence. We say that a coding $\alpha$ is periodic (rational) if there exists a natural number $\mathrm{p}$ such that $\alpha_{k+p}=\alpha_{k}$ for all $k \in \mathcal{N}$, and we denote the coding $\alpha$ by $\alpha=\mathcal{P}\left(\alpha_{0} \ldots \alpha_{p-1}\right)$ where $\mathcal{P}$ is the periodic concatenation operation. In fact for an invertible piecewise isometry, a rational coding is always periodic. A point $z \in \pi(M) \backslash \mathcal{E}_{-}$is a rational point if its coding is periodic and let $\mathcal{R}$ be the set of all rational points in $\pi(M)$, and $\mathcal{J}$ be the set of all irrational points in $\pi(M)$. Denote by $D(\alpha)$ the set of all points with the coding $\alpha$ (when $\alpha$ is infinite) or starting at $\alpha$ (when $\alpha$ is finite), 
and $D(\alpha)$ is called the $\alpha$-cell. Finally, we say a map $f_{\alpha}=f_{\alpha_{n-1}} \circ \ldots \circ f_{\alpha_{0}}$ is admissible if the sequence $\alpha$ is admissible.

Obviosly, for any non-forward-singular point, we can well define a unique coding, while, for a forward singular point $\mathrm{z}$, we cannot define a coding as above since $f^{n}(z)$ belongs to $\partial M$ for some natural number n. For convenience of discussion, we must generalize the coding map such that it can be well defined on the forward singular set.

Definition 1. For a forward singular point $z \in \mathcal{E}_{-}$, we say a sequence $\alpha$ is an essential coding of $\mathrm{z}$ if for all $n \in \mathbb{N}$ and for all $\varepsilon>0$, there exists a point $z^{\prime} \in B(z, \varepsilon) \backslash \mathcal{E}_{-}(n)$ such that $\left[\mathcal{L}\left(z^{\prime}\right)\right]_{j}=\alpha_{j}, \quad j=0, \ldots, n$.

In fact, an essential coding corresponding to a forward singular point is set-valued. Then we can extend the definition of coding map onto the entire phase space $\pi(M), \overline{\mathcal{L}}: \pi(M) \mapsto \sum(m)$ as follows

$$
\overline{\mathcal{L}}(z)= \begin{cases}\mathcal{L}(z) & \text { if } z \notin \mathcal{E} \\ \{\text { all of the essential coding of } z\} & \text { if } z \in \mathcal{E}_{-}\end{cases}
$$

. If $z \in \mathcal{E}_{-}$and $\alpha=\alpha_{0} \alpha_{1} \ldots$ is one of its essential coding, we call the iteration $f^{n}(z)=f_{\alpha_{n-1}} \circ \ldots \circ f_{\alpha_{1}} \circ f_{\alpha_{0}}$ an essential iteration.

Theorem 2. For a forward singular point $z$, there must exist an essential coding. [8]

In the following, we give an equivalent proposition for essential codings.

Theorem 3. An infinite sequence $\alpha$ is one of the essential codings of a forward singular point $z$ if and only if for arbitrary small $\varepsilon>0$ there exists a point $z^{\prime} \in B(z, \varepsilon) \cap \pi(M) \backslash \mathcal{E}_{-}$such that

$$
d\left(\alpha, \mathcal{L}\left(z^{\prime}\right)\right)
$$

where $d(\alpha, \beta)$ represents the distance of two infinite sequence $\alpha$ and $\beta$.

\section{Main definition and results}

In this section we define a system of planar piecewise isometric systems and the coding map of this systems. Some definition and proposition are also given. From these proposition, we can understand the relation among singular sets, exceptional sets, irrational sets, and at the end we show that the necessary and sufficient condition of exists rational coding is $\mathcal{F} \neq \pi(M)$. 
Definition 4. Let $\mathrm{M}$ is a set and $\mathrm{P}(\mathrm{M})$ is the power set of $\mathrm{M}$ then we define a partion function $\rho$ on $\mathrm{M}$ as follows

$$
\rho: \mathbb{N} \mapsto P(P(M))
$$

I: $\quad \# \rho(t)=m_{t}<\infty \quad ; \quad$ for all $t \in \mathbb{N}$

II: $\bigcup_{M_{i}^{t} \in \rho(t)} M_{i}^{t}=M \quad ; \quad$ for all $t \in \mathbb{N}$

III: $\quad M_{i}^{t} \cap M_{j}^{t}=\emptyset \quad ; \quad$ for all $t \in \mathbb{N}$ and for all $i, j \in\left\{1,2, \ldots, m_{t}\right\}$

Let $\mathrm{M}$ is a bounded subset of $\mathbb{R}^{n}$ and $\rho$ is a partition function on $\mathrm{M}$ with following special conditions

I: $\quad m_{t}=m_{t^{\prime}}=m \quad ; \quad$ for $t \neq t^{\prime} \quad$ and for all $t, t^{\prime} \in \mathbb{N}$

II: $\quad \rho(t)=\left\{M_{1}^{t}, M_{2}^{t}, \ldots, M_{m}^{t}\right\}$ for all $t \in \mathbb{N}$ is a partition of the compact region $\pi(M)=\bigcup_{k=1}^{m} \bar{M}_{k}^{t}$ where $\left\{M_{k}^{t}\right\}$ are disjoint open polygons which are called the partition atoms.

III: $\quad \pi(M)=\bigcup_{M_{k}^{t} \in \rho(t)} \bar{M}_{k}^{t}=\bigcup_{M_{k}^{t^{\prime}} \in \rho\left(t^{\prime}\right)} \bar{M}_{k}^{t^{\prime}} \quad ; \quad$ for $t \neq t^{\prime}$ and for all $t, t^{\prime} \in \mathbb{N}$

Definition 5. Let $\mathrm{M}$ is a bounded subset of $\mathbb{R}^{n}$ and $\rho$ is a partition function on $\mathrm{M}$ with special condition, and for all $t \in \mathbb{N},\left(f^{t}, M\right)$ is a piecewise isometric system, so a pair $\left(M,\left\{f^{t} \mid t \in \mathbb{N}\right\}\right)$ is called a system of planar piecewise isometric systems.

Definition 6. Let $\left(M,\left\{f^{t} \mid t \in \mathbb{N}\right\}\right)$ is a system of planar piecewise isometric systems with partition function $\rho$ then we define the phase space $\pi$ as follows:

$$
\pi(M)=\bigcup_{M_{k}^{t} \in \rho(t)} \bar{M}_{k}^{t}
$$

Definition 7. Let $\left(M,\left\{f^{t} \mid t \in \mathbb{N}\right\}\right)$ is a system of planar piecewise isometric systems with partition function $\rho$. The $\partial^{t} M=\bigcup_{M_{i}^{t}, M_{j}^{t} \in \rho(t)} \bar{M}_{i}^{t} \cap \bar{M}_{j}^{t}$ for all $t \in \mathbb{N}$ is called t-discontinuty line segment (t-boundry of phase space).

Definition 8. Let $\left(M,\left\{f^{t} \mid t \in \mathbb{N}\right\}\right)$ is a system of planar piecewise isometric systems with partition function $\rho$.

Let

$\mathcal{E}_{-}^{t}(n)=\bigcup_{k=0}^{n}\left(f^{t}\right)^{-k}\left(\partial^{t} M\right) \quad ; \quad$ for all $t \in \mathbb{N}, n \in \mathbb{N} \cup\{0\}$

$\mathcal{E}_{+}^{t}(n)=\bigcup_{k=1}^{n}\left(f^{t}\right)^{k}\left(\partial^{t} M\right) \quad ; \quad$ for all $t \in \mathbb{N}, n \in \mathbb{N}$

we called $\mathcal{E}_{-}^{t}=\lim _{n \rightarrow \infty} \mathcal{E}_{-}^{t}(n)\left(\mathcal{E}_{-}^{t}=\bigcup_{k=0}^{\infty}\left(f^{t}\right)^{-k}\left(\partial^{t} M\right)\right)$ the t-forward singular set, and $\mathcal{E}_{+}^{t}=\lim _{n \rightarrow \infty} \mathcal{E}_{+}^{t}(n)\left(\mathcal{E}_{+}^{t}=\bigcup_{k=1}^{\infty}\left(f^{t}\right)^{k}\left(\partial^{t} M\right)\right)$ the t-backward 
singular set , and $\mathcal{E}^{t}=\mathcal{E}_{-}^{t} \cup \mathcal{E}_{+}^{t}$ the t-sigular set we call $\mathcal{E}^{\prime}=\bigcap_{t \in \mathbb{N}} \mathcal{E}^{t}$ the singular set with variant behavior and $\mathcal{E}_{-}=\bigcap_{t \in \mathbb{N}} \mathcal{E}_{-}^{t}$ the forward singular set and $\mathcal{E}_{+}=\bigcap_{t \in \mathbb{N}} \mathcal{E}_{+}^{t}$ the backward singular set and $\mathcal{E}=\mathcal{E}_{-} \cup \mathcal{E}_{+}$the singular set with stable behavior.

lemma 9. Let $\left(M,\left\{f^{t} \mid t \in \mathbb{N}\right\}\right)$ is a system of planar piecewise isometric systems with partition function $\rho$.

Let

$\mathcal{E}_{-}(n)=\bigcap_{t \in \mathbb{N}} \mathcal{E}_{-}^{t}(n) \quad ; \quad$ for all $t \in \mathbb{N}$

$\mathcal{E}_{+}(n)=\bigcap_{t \in \mathbb{N}} \mathcal{E}_{+}^{t}(n) \quad ; \quad$ for all $t \in \mathbb{N}$

Then

1) $\mathcal{E}_{-}=\lim _{n \rightarrow \infty} \mathcal{E}_{-}(n)$

2) $\mathcal{E}_{+}=\lim _{n \rightarrow \infty} \mathcal{E}_{+}(n)$

Definition 10. Let $\left(M,\left\{f^{t} \mid t \in \mathbb{N}\right\}\right)$ is a system of planar piecewise isometric systems with partition function $\rho$, we called

$$
\mathcal{F}^{t}=\left\{z \in M \mid i n f_{n \in \mathbb{Z}} d\left(\left(f^{t}\right)^{n}(z), \partial^{t} M\right)=0\right\}
$$

the t-exeptional set, where $\mathrm{d}(.,$.$) represents the Euclidean metric and we$ define the exceptional set $\mathcal{F}$ as follows,

$$
\mathcal{F}=\bigcap_{t \in \mathbb{N}} \mathcal{F}^{t}
$$

lemma 11. Let $\left(M,\left\{f^{t} \mid t \in \mathbb{N}\right\}\right)$ is a system of planar piecewise isometric systems with partition function $\rho$ then

$$
\begin{aligned}
& \mathcal{E} \subseteq \mathcal{E}^{\prime} \subseteq \mathcal{F} \\
& \overline{\mathcal{E}} \subseteq \overline{\mathcal{E}}^{\prime} \subseteq \mathcal{F}
\end{aligned}
$$

Definition 12. Let $\left(M,\left\{f^{t} \mid t \in \mathbb{N}\right\}\right)$ is a system of planar piecewise isometric systems with partition function $\rho$ and for all $t \in \mathbb{N}, \mathcal{L}^{t}$ is a coding map define in preliminary for piecewise isometric system $\left(f^{t}, M\right)$ with partition $\rho(t)$ and sequence of isometric maps $f^{t}=\left\{f_{1}^{t}, \ldots, f_{m}^{t}\right\}$ so we define the coding map $\mathcal{L}: \pi(M) \backslash \mathcal{E}_{-} \mapsto \sum(m)$ as follows,

$$
\mathcal{L}(z)=\left\{\begin{array}{lll}
\mathcal{L}_{t}(z) & \text { if } z \notin \mathcal{F} & \left(\text { Where } t \text { is the smallest } t \in \mathbb{N} \text { such that } z \notin \mathcal{F}^{t}\right) \\
\mathcal{L}_{t}(z) & \text { if } z \in \mathcal{F} \backslash \mathcal{E}_{-} & \left(\text {Where } t \text { is the smallest } t \in \mathbb{N} \text { such that } z \notin \mathcal{E}_{-}\right)
\end{array}\right.
$$


Obviously for any non-forward-singular point we can well define a unique coding. For convenience of discussion we must generalize the coding map such that it can be well define on the forward singular set. Let $z \in \mathcal{E}_{-}$now we define the coding of $\mathrm{z}$ as follows,

$$
\mathcal{L}(z)=\mathcal{L}_{t}(z) \Leftrightarrow \forall \delta>0 \quad \exists z_{0} \in B(z, \delta) \backslash \mathcal{E}_{-} \text {such that } \mathcal{L}\left(z_{0}\right)=\mathcal{L}_{t}\left(z_{0}\right)
$$

In this time we say $\mathcal{L}(z)=\mathcal{L}_{t}(z)$ is the essentioal coding of point $\mathrm{z}$, where $\mathcal{L}_{t}(z)$ is the t-essential coding of point $\mathrm{z}$ define in Definition 1 , if $z \in \mathcal{E}_{-}^{t}$.

Definition 13. Let $z \in \pi(M)$ and $\mathcal{L}(z)=\mathcal{L}_{t}(z)$ is the essential coding of point $\mathrm{z}$ then we say $\mathrm{t}$ is the essential time of point $\mathrm{z}$.

lemma 14. The set $\mathcal{E}^{\prime}$ has zero lebesgue measure.

Corollary 15. The set $\mathcal{E}$ has zero lebesgue measur.

Theorem 16. For a forward singular point $z$ there must exist an essential coding.

Proof. Let $z \in \mathcal{E}_{-}$. At first we know that for $\delta>0$ the set $B(z, \delta) \backslash \mathcal{E}_{-}$ is nonempty, and the set $\mathcal{E}_{-}$has zero lebesgue measur. We show that there exist $t \in \mathbb{N}$ such that, for all $\delta>0$ there exist $z_{0} \in B(z, \delta) \backslash \mathcal{E}_{-}$such that $\mathcal{L}\left(z_{0}\right)=\mathcal{L}_{t}\left(z_{0}\right)$.

Proof by cotradiction. Let for all $t \in \mathbb{N}$ there exist $\delta_{t}>0$ such that for all $z_{0} \in B\left(z, \delta_{t}\right) \backslash \mathcal{E}_{-}$there exist $t^{\prime}<t$ such that $z_{0} \notin \mathcal{E}_{-}^{t}$. Let $t \in \mathbb{N}$ is fixed so there exist $\delta_{0}>0$ such that for all $z_{0} \in B\left(z, \delta_{0}\right) \backslash \mathcal{E}_{-}$there exist $t^{\prime}<t$ such that $z_{0} \notin \mathcal{E}_{-}^{t^{\prime}}$ and $\mathcal{L}\left(z_{0}\right)=\mathcal{L}_{t \prime}\left(z_{0}\right)$. This means $t^{\prime}$ is the smallest time that $z_{0} \notin \mathcal{E}_{-}^{t}$ let $K=\{1,2, \ldots, t-1\}$. Then there exist $t_{-}^{\prime}<t$ such that $t_{0}^{\prime}$ is the essential time for infinit point in $B(z, \delta) \backslash \mathcal{E}_{-}$. If $t_{0}^{\prime}$ is the unique element of $\mathrm{K}$ such that $t_{0}^{\prime}$ is the essential time for infinit point in $B(z, \delta) \backslash \mathcal{E}_{-}$so for all $\delta^{\prime}>0$ there exist $z_{0} \in B\left(z, \delta^{\prime}\right) \backslash \mathcal{E}_{-}$such that $\mathcal{L}\left(z_{0}\right)=\mathcal{L}_{t \prime}\left(z_{0}\right)$. This is a contradiction. If $t^{\prime}$ is the unique element of $\mathrm{K}$ such that $t^{\prime}$ is the essential time for infinite point in $B(z, \delta) \backslash \mathcal{E}_{-}$now with this assume we have the following assertions. A.There exist $t_{0}^{\prime}$ and there exist the sequence $z_{n}$ in $B\left(z, \delta_{t}\right) \backslash \mathcal{E}_{-}$such that the sequence $z_{n}$ limited to $\mathrm{z}$ and for all $n \in \mathbb{N}, t_{0}^{\prime}$ is the essential time for $z_{n}$.

Proof of A. Proof by contradiction. Let for all $t_{i} \in K$ we dont have any sequence $z_{n}$ such that limited to z. On the other word let $K_{i}$ be the set of all points $z \in \pi(M)$ with the essential time $t_{i}$. Then for all $t_{i}<t$ here exist $\delta_{i}$ such that $B(z, \delta) \cap K_{i}=\emptyset$. Let $\delta_{0}=\min \left\{\delta_{1}, \ldots, \delta_{t}\right\}$ then we have

$$
B\left(z, \delta_{0}\right) \bigcap\left(\bigcup_{i=1}^{t-1} K_{i}\right)=\emptyset
$$


Therefore $\mathrm{t}$ is the essential time for all $z_{0} \in B(z . \delta) \mathcal{E}_{-}$, and this is a contradiction.

Definition 17. Let $\left(M,\left\{f^{t} \mid t \in \mathbb{N}\right\}\right)$ is a system of planar piecewise isometric systems with partition function $\rho$. Then for all $t \in \mathbb{N}, \mathcal{J}^{t}$ is the set of all irrational points in $\left(M, f^{t}\right)$, and $\mathcal{R}^{t}$ is the set of all rational points in $\left(M, f^{t}\right)$, and let $\mathcal{J}$ be the set of all irrational points in $\left(M,\left\{f^{t} \mid t \in \mathbb{N}\right\}\right)$, and $\mathcal{R}$ be the set of all rational points in $\left(M,\left\{f^{t} \mid t \in \mathbb{N}\right\}\right)$.

Corollary 18. For a system of planar piecewise isometric systems we have;

$$
\begin{gathered}
\mathcal{J}=\bigcup_{t \in \mathbb{N}} \mathcal{J}^{t} \\
\mathcal{R}=\bigcup_{t \in \mathbb{N}} \mathcal{R}^{t} \\
\mathcal{J} \subseteq \mathcal{F}
\end{gathered}
$$

Theorem 19. Given a system of planar piecewise isometric dynamical systems $\left(M,\left\{f^{t} \mid t \in \mathbb{N}\right\}\right)$ with partition function $\rho$, and suppose the phase space has positive bounded Lebesgue measure, then there exists an admissible rational coding if and only if $\mathcal{F} \neq \pi(M)$.

Proof. Let the point $z \in \pi(M)$ be a point with admissible rational coding, then there exist $t \in \mathbb{N}$ such that $\mathrm{t}$ is the essential time of point $\mathrm{z}$, and $\mathcal{L}_{t}(z)$ is an admissible rational coding, so with [8] the set $\mathcal{E}_{-}^{t}$ is not dense in the phase space. On the other hand we have $\overline{\mathcal{E}_{-}^{t}}=\mathcal{F}^{t}$ therefore $\mathcal{F} \subseteq \mathcal{F}^{t} \neq \pi(M)$. Conversely. Let $\mathcal{F} \neq \pi(M)$ and $z \in \pi(M) \backslash \mathcal{F}$ then there exist $t \in \mathbb{N}$ such that $z \notin \mathcal{F}^{t}$ and $\mathrm{t}$ is the essential time of the point $\mathrm{z}$, and by we know that $\mathcal{J}^{t} \subseteq \mathcal{F}$, therefore the point $\mathrm{z}$ has an admissible rational coding.

Corollary 20. For a system of planar piecewise isometric dynamical systems $\left(M,\left\{f^{t} \mid t \in \mathbb{N}\right\}\right)$ if the equalities $\mathcal{F}=\pi(M)$ hold, then all admissible points are irrational, i.e., $\mathcal{J}=\pi(M)$.

Proof. Suppose $\mathcal{F}=\pi(M)$, and we know that $\mathcal{F}=\bigcap_{t \in \mathbb{N}} \mathcal{F}^{t}$ then for all $t \in \mathbb{N}$ the equalities $\mathcal{F}^{t}=\pi(M)$ hold. So with the proof is completed. 


\section{4. conclusions}

the main discussed in this paper is system of planar piecewise isometric dynamical systems. we could introduced this system by use a partition function and by introduce a coding map we investigate the important sets including the singular sets, the exceptional set, ... .

The main part of thise paper is difinition and extention the coding map for a system of planar piecewise isometric systems. then we illustrate in the theorem 15 for a forwared singular point there must exists an essential coding.

In the future we can investigate the more proposition of these systems like that rational and irrational coding, chaos ,... .

\section{References}

[1] A.C.Davies, Geometrical analysis of digital filters overflow oscillation. Proc. IEEE Symp. Circuits and Systems, San Diego, pages 256-259 (1992).

[2] A.C.Davies, Nonlinear oscillations and chaos from digital filter overflow. Phil. Trans. R. Soc. Lond. A 353:85-99 (1995).

[3] A.Goetz, Dynamics of piecewise Isometries, (1999).

[4] A.Goetz, Dynamics of a pecewise rotation. Continuous and Discrete Dynamical systems 4:593-608 (1998).

[5] A.Goetz, Perturbation of 8-attractors and births of satellite systems, Intr. J. Bifu. Chaos 8, 19-37 (1998).

[6] A.Goetz, Sofic subshifts and piecewise isometric systems. Ergodic Theory and Dyn. Sys., 19 (1999) 1485-1501.

[7] C.Boldrigini, M.Keane, and F.Marchetti, Billiards in polygons. Ann. of Probab., 4:532540 (1976).

[8] E.Gutkin and H. Haydn, Topological entropy of generalized polygon exchanges. Bull. Amer. Math. Soc. 32:50-57 (1995).

[9] E.Gutkin and N.Haydn, topological entropy of generalized polygon exchanges. Ergodic Theory and Dynamical Systems, 17:849-867 (1997).

[10] E.Gutkin, Billiards in polygons: survey of recent results. J. Stat. Phys., 83:7-26 (1996).

[11] G.A.Galperin, Two constructive sufficent condition for aperiodicity of interval exchange. Theoretical and Applied Problems of Optimization, 176:8-16 (1985).

[12] H.Haller, Rectangel exchange trnsformations. Mathematik, 3:215-232 (1981).

[13] H.Masur, Interval exchange tranformations and measured foliations. Annals of Mathematics,115:169-200 (1982).

[14] J.Buzzi, Piecewise isometries have zero topological entropy, Ergodic Theory Dynam. Systems 21J. Buzzi, Piecewise isometries have zero topological entropy, Ergodic Theory Dynam. Systems 21: 13711377 (2001). 
[15] L.O.Chua and T.Lin, Chaos and fractals from third-order digital filters. Internat. J. Circuit Theory Appl. 18(3):241-255 (1990).

[16] L.O.Chua and T. Lin, Chaos in digital filters. IEEE Trans. Circuits and Systems, 35(6):648-658 (1988).

[17] Lj.Kocarev, C.W.Wu, and L.O.Chua, Complex behavior in digital filters with overflow nonlinearity: analytical results. IEEE Trans. Circuits and Systems II: Analog and digital signal processing, (43):234-246 (1996).

[18] M.Mendes, M.Nicol, Periodicity and recurrence in piecewise rotations of Euclidean spaces, Internat. J. Bifur. Chaos 14: 23532361 (2004).

[19] M.Mendes, Quasi-invariant attractors of piecewise isometric systems, Discrete Contin. Dyn. Syst. 9: 323338 (2003).

[20] M.Mends, Stability of periodic points in piecewise isometries of Euclidean spance, Ergodic Theory Dynam. Systems 27: 183197 (2007).

[21] M.Boshernitzan and I.Kornfeld, Interval translations maping. Ergodic Theory And Dynamical Systems, 15:821-831 (1995).

[22] M.Boshernitzan, Acondition for minimal interval exchange maps to be uniquely ergodic. Duke Mathematical Journal, 52:723-752 (1985).

[23] M.J.Ogorzalek, Complex behavior in digital filters. Internat. J. Bifur. Chaos Appl. Sci. Engrg., 2(1):11-29 (1992).

[24] M.Keane, Interval exchange transformations. Mathematische Zeitung, 141:25-31 (1975).

[25] P.Arnoux, D. Orstein, and B. Weiss, Cutting and stacking, interval exchanges, and geometric models. Israel J. Maht, pages 160-168 (1985).

[26] P.Ashwin and X.-C. Fu, Tangencies in invariant disk packings for certain planar piecewise isometries are rare. Dynamical Systems, 16: 333-345 (2001).

[27] P.Ashwin and X.-C.Fu, On the geometry orientation preserving planar piecewise isometries, (2002).

[28] X.-C.fu and P.Ashwin, symbolic analysis for some planar piecewise linear maps(2008)

[29] P.Ashwin, W.Chambers, and G.Petrov, Lossless digital fillter overflow oscillations; approximation of invariant fractals. Internat. J. Bifur. Chaos Appl.Sci. Engrg., 7:2603-2610 (1997).

[30] P.Ashwin, X.-C.Fu and J.H.B.Deane, Properties of the invariant disk packing in a model bandpass sigma-delta modulator. To appear in Int. J. of Bif. and Chaos, Vol.13 (2003).

[31] P.Ashwin, Non-smooth invariant circles in digital overflow oscillations. Proceedings of NDE96: fourth international workshop on Nonlinear Dynamics of Electronic Systems, Seville, Spain (1996).

[32] R.Adler, B.Kitchens and C.Tresser, Dynamics of nonergodic piecewise affine maps of the torus. Preprint,1999

[33] R.-Z. Yu, X.-C.Fu, K.-M.Wang, Z.-H.Chen, Dynamical behaviors and codings of invertible planar piecewise isometric systems, Nonlinear Anal. 72: 35753582 (2010).

[34] W.Veech, Gauss measures for transfomation on the space of interval exchange maps. Annals of Mathematics, 115:201-242 (1982).

[35] W.Veech, Interval exchange transformations. J. D,Analyse Math. 33:222-272 (1978). 